\title{
The effect of diet and nutritional elements on gut microbiota
}

\author{
Ahmet Akçakaya ${ }^{1}$, Funda İfakat Tengiz ${ }^{2}$ (D) \\ ${ }^{1}$ Izmir Katip Çelebi University Faculty of Medicine, Izmir, Turkey \\ ${ }^{2}$ Department of Medical Education, Izmir Katip Çelebi University Faculty of Medicine, Izmir, Turkey
}

\begin{abstract}
The human gut microbiota is a structure that consists of microorganisms such as bacteria, viruses, fungi, and protozoa. Gut microbiota plays a role in many biochemical events. For this reason, it is called a metabolic organ and contains more than 1000 microorganisms. Microbiota is closely related to diet and microbiota structure varies from person to person due to differences in diet. Microbiota is also affected by various factors such as breastfeeding, method of birth, the condition of the mother's intestinal microbiota, and environmental factors. This study aims to demonstrate the structure and development of microbiota and its association with diet and nutrients.

Keywords: Diet, microbiota, prebiotic.
\end{abstract}

Gut microbiota is a structure with very important functions that consists of microorganisms such as bacteria, viruses, fungi, and protozoa within the gastrointestinal tract. ${ }^{[1]}$ It contains more than 1,000 microorganisms. These species can be mutualist, commensal, or pathogenic. ${ }^{[1]}$ There are six bacterial groups in a healthy individual's microbiota:

1. Firmicutes (includes Gram positive genera such as Clostridium, Ruminococcus, Roseburia)

2. Bacteroidetes (includes Gram negative genera such as Bacteroides, Prevotella)

3. Proteobacteria (includes Gram negative genera such as Enterobacteriaceae)

4. Fusobacterium

5. Actinobacteria (includes Gram positive Bifidobacterium genus)

6. Verrucomicrobia (includes genus such as Akkermansia $)^{[2]}$
The numbers of these groups may vary depending on various factors, while the Gram-positive Firmicutes and Gram-negative Bacteroidetes groups are most abundant. ${ }^{[1,2]}$ Gut microbiota takes part in physiological, metabolic, immunological, and neural functions, and therefore considered a metabolic organ. ${ }^{[2]}$ There are great differences in the diversity of microorganisms from the esophagus to the rectum. While there are 10 colonies of microorganisms in the esophagus and stomach, there are 10 to 12 colonies in the colon and distal intestine. ${ }^{[3]}$ Healthy gut microbiota strengthens metabolism in circumstances of infection and inflammation, increases resistance to cancers and autoimmune diseases, and plays a role in endocrine signal and brain functions (gutbrain axis). ${ }^{[3]}$

It is thought that gut microbiota is related to various diseases as it performs important biochemical events. ${ }^{[2]}$ Beneficial bacteria are involved in biochemical processes of vitamins,

Received: October 25, 2019 Accepted: December 05, 2019 Published online: April 15, 2020

Correspondence: Ahmet Akçakaya. İzmir Katip Çelebi Üniversitesi Tıp Fakültesi, 35620 Çiğli, İzmir, Türkiye. Tel: +90553- 2081122 e-mail: ahmet.akcakaya99@gmail.com 
short-chain fatty acids (SCFAs) synthesis, conjugated linoleic acid (CLA) production, amino acid synthesis, bile acid biotransformation, fermentation of undigested foods, and immunomodulation. When the "beneficial/ harmful" ratio of the bacteria in flora decreases, pathology called microbial dysbiosis occurs. Microbial dysbiosis has been associated with many diseases such as allergies, cancer, lupus, asthma, multiple sclerosis, Parkinson's syndrome, gluten enteropathy, obesity, diabetes, inflammatory bowel disease, and cardiovascular diseases. ${ }^{[2]}$

Recent studies have discovered that the infant gastrointestinal system, thought to be sterile until birth, is actually unsterile and that microbiota development begins in the womb. ${ }^{[1]}$ This prenatal microorganism transmission is provided through the placenta. ${ }^{[4]}$ In the first days of life after birth, the baby's rate of exposure to bacteria increases and the microbiota develops with the transition to additional nutrients. ${ }^{[1]}$ At the age of 2-3 years, the microbiota resembles the adult microbiota both structurally and functionally. ${ }^{[1]}$ Factors affecting microbiota development include mode of delivery, breast milk intake, exposure to bacteria, use of antibiotics and probiotics, and nutrition. After the microbiota develops, its structure is affected by similar factors. ${ }^{[1]}$

\section{EFFECT OF DIET ON MICROBIOTA}

Type of diet is an important factor in microbiota structure and health. Since diet types varies according to geography, different microbiota structures in different geographical regions draw attention. The amount of residue in the diet is directly related to microbiota health. The content of protein and fat in the diet also plays an important role in the microbiota structure. ${ }^{[1]}$

Traditional Mediterranean diet consists of unsaturated fat consumption (olive oil), excessive legume consumption, high consumption of unrefined grain (bread), low consumption of meat products, moderate consumption of dairy products, and moderate consumption of alcohol (red wine). Although fish consumption is one of the important features of this diet, consumption is parallel to proximity to the sea. ${ }^{[2]}$ Some studies have shown that the typical Mediterranean diet improves obesity, lipid profile, and inflammation. This diet may cause an increase in Lactobacillus,
Bifidobacterium and Prevotella, and a decrease in Clostridium, ${ }^{[2]}$ as wells as high levels of fecal SCFAs. ${ }^{[1]}$ The Mediterranean diet is generally considered a balanced diet.

Residue consumption is an important element for cultivating microorganisms and therefore microbiota health. The typical Western diet cannot properly sustain microbiota since it is low in residue content and rich in animal proteins and fat, while the rural African diet is rich in residue content and vegetable proteins, providing microbiota with the necessary residue. In a study conducted by De Fillipo et al., ${ }^{[5]}$ the microbiota structure of African children who were fed a diet rich in residue and vegetable protein was compared to the microbiota structure of Italian children of the same age who were fed a low-fiber typical Western diet rich in animal protein and fat. The abundance and diversity of microbiota was found to be higher in African children. Meanwhile, the quantity of Firmicutes and Proteobacteria was high in the microbiota of Italian children, while Prevotella, Xylanibacterium and Treponema were found higher in African children. ${ }^{[1]}$

Ten healthy volunteers in America were put on a gluten-free diet for 30 days. According to the gut microbiota of these individuals, the number of beneficial bacteria such as Bifidobacterium, Lactobacillus and Bifidobacterium Longum were shown to decrease while the number of pathogenic species such as Escherichia coli (E. coli) and Enterobacteriaceae had increased parallel to the decrease in polysaccharide intake. ${ }^{[2,6]}$ Since polysaccharide intake is very limited with this diet, limited carbohydrate compounds reach the colon and saccharolytic fermentation cannot be

Table 1. The effect of certain diets on microbiota ${ }^{[2]}$

\begin{tabular}{lccc}
\hline & \multicolumn{3}{c}{ Diet } \\
\cline { 2 - 4 } & Western & Mediterranean & Gluten-free \\
\hline Total bacteria & $\downarrow$ & $\uparrow$ & $\downarrow$ \\
Bifidobacteria & $\downarrow$ & $\uparrow$ & $\downarrow$ \\
Lactobacilliare & $\downarrow$ & $\uparrow$ & $\downarrow$ \\
Prevotella & & $\uparrow$ & $\downarrow$ \\
Eubacteria & $\downarrow$ & $\uparrow$ & $\downarrow$ \\
Roseburia & & $\uparrow$ & $\downarrow$ \\
Bacteroides & $\uparrow$ & $\uparrow$ & \\
Enterobacteria & $\uparrow$ & $\uparrow$ & $\uparrow$ \\
\hline
\end{tabular}


adequately performed. Therefore, both SCFAs cannot be formed and the number of probiotic bacteria producing butyrate decreases while the number of pathogens increases. ${ }^{[1]}$

\section{MICROBIOTA DEVELOPMENT AND THE SIGNIFICANCE OF BREAST MILK}

It was previously believed that amniotic fluid and the fetus were completely sterile before birth, and that the baby was exposed to various bacteria during and after birth. ${ }^{[4]}$ According to the results of research, however, it was revealed that the baby encountered the mother's intestinal microbiota in the fetal period via the placenta. ${ }^{[4]}$

Various changes occur in the mother from the start of pregnancy. Certain types of bacteria were found in breast milk in the late stages of pregnancy. Studies revealed that bacteria in the mother's gut flora are transported to the mother's milk glands through intestinal immune cells during breastfeeding. ${ }^{[4]}$

Maternal diet greatly influences maternal microbiota, and hence the baby's microbiota. ${ }^{[4]}$ Since the use of antibiotics and probiotics significantly affects the mother's microbiota, the use of these agents during pregnancy is extremely impactful to the baby's microbiota. ${ }^{[4]}$

The baby's method of delivery, just like breast milk, is also an important factor in the baby's microbiota. Due to physiological stress and lack of certain hormones, the passage of microbiota into breast milk is impaired in mothers who give birth by cesarean section. ${ }^{[4]}$ Because babies born with cesarean birth do not come into contact with the mother's vaginal bacteria, microbiota development is negatively affected and these babies only encounter the mother's dermal microbiota during delivery. This phenomenon is thought to be the reason for the high prevalence of allergy and asthma in babies born by cesarean section. ${ }^{[4]}$

While breast milk contains macro- and micronutrients necessary for feeding the baby, it also contains probiotics and prebiotics to support the development of the baby's microbiota. ${ }^{[4]}$ Breast milk has its own microbiota, which contains more than 200 bacterial phylotypes. ${ }^{[4]}$ In general, 50\% of breast milk microbiota is common among all mothers, while $50 \%$ varies according to maternal and environmental factors. ${ }^{[4]}$ Among these common bacteria, the most abundant in breast milk is Staphylococcus and Streptococcus. ${ }^{[4]}$ The factors that determine the $50 \%$ varying portion of maternal microbiota are the mother's health, diet, immunological status, mode of delivery, antibiotic use, and obesity.

Breast milk microbiota is directly related to the mother's own gut microbiota. Therefore, the healthier the mother's gut microbiota, the healthier the baby's microbiota development will be. ${ }^{[4]}$

The first three months of microbiota development are vital to the baby. The baby's encounter with vaginal, dermal, and fecal microbiota of the mother with birth initially

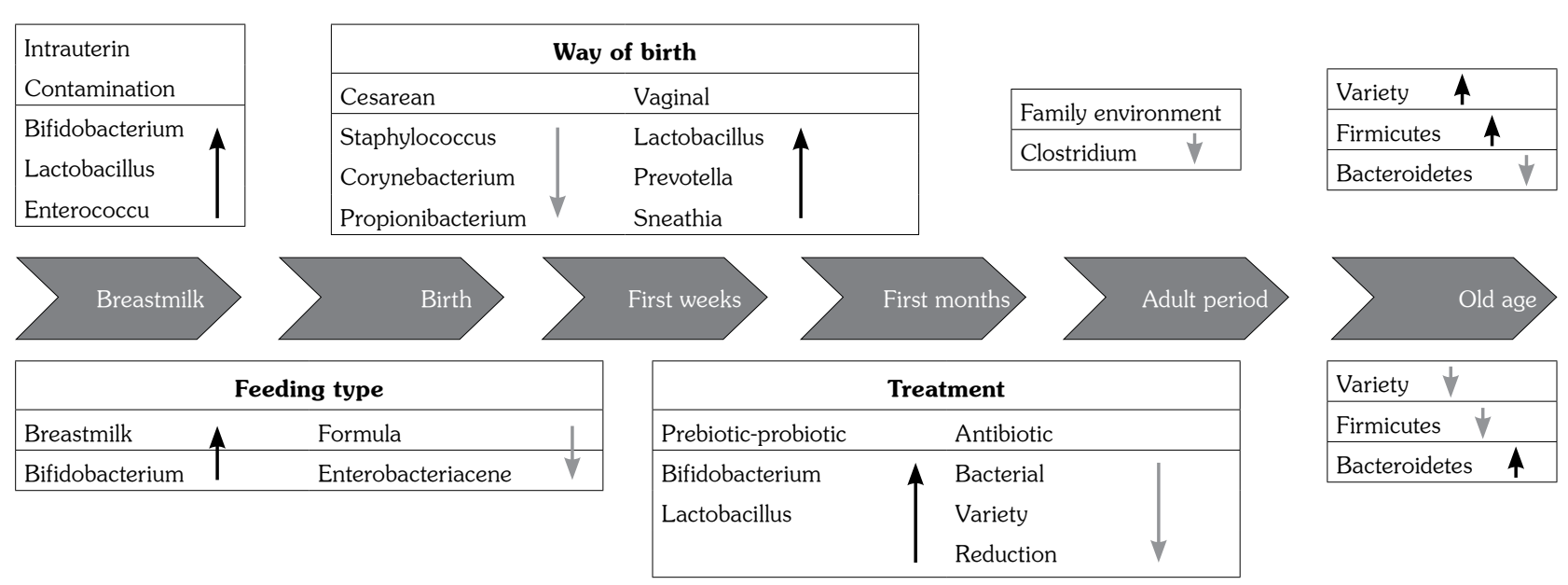

Figure 1. Intestinal microbiota development. ${ }^{[2]}$ 
establishes colonies of bacteria belonging to these microbiota in the baby's gastrointestinal tract (E. coli, Staphylococcus, Streptococcus) and provide the conditions for bacteria passed from breast milk that can only be colonized in the anaerobic environment in the child's intestinal cavity (Bacteroidetes and Bifidobacteria) ${ }^{[4]}$ Afterwards, these Bacteroidetes and Bifidobacteria become dominant among other species. On the other hand, in babies who are not breastfeeding, the variety of bacteria is high while the number of Bifidobacteria is low. ${ }^{[4]}$

Another useful element of breast milk for microbiota is oligosaccharides. Babies do not have enzymes that can digest the glycan in milk. Therefore, glycan descends into the intestine and contributes to cultivating microbiota. ${ }^{[4]}$ Lactoferrin in breast milk also has a prebiotic feature. ${ }^{[4]}$

Since breast milk provides both the bacteria necessary for the baby microbiota, it is probiotic, and also contains compounds such as oligosaccharides and lactoferrin which help sustain bacteria, it is also prebiotic. ${ }^{[4]}$

\section{THE EFFECT OF NUTRITIONAL ELEMENTS ON MICROBIOTA}

\section{1) Carbohydrates}

Carbohydrates are an energy source for colon microbiota. Non-digestible carbohydrates including cellulose, starch, and non-starch polysaccharides, as well as normally digestible carbohydrates that reach the colon by escaping digestion are used in energy production by fermentation. ${ }^{[1]}$ As a result of this fermentation, SCFAs and gases are formed. ${ }^{[1]}$ These SCFAs formed are absorbed in the colon and perform important functions. Some of them include:
1. Butyrate: increases apoptosis of cancer cells in the colon (anticarcinogenic effect), ${ }^{[1]}$ an energy source for colon epithelium. ${ }^{[2,6]}$

2. Propionate: an energy source for epithelial cells that is involved in gluconeogenesis, important in regulating the sensation of hunger-satiety. ${ }^{[1]}$

3. Acetate: the most abundant SCFA in the intestine; plays a role in lipogenesis; important in hunger-satiety regulation.

Nourishment of the gut microbiota depends on the amount of carbohydrates that reach the colon without being digested, rather than the total amount of carbohydrates obtained by diet. ${ }^{[2,6]}$ Here too, the concepts of residue and fiber are important. Fermentation responses of gut microbiota vary according to the types of residues and fibers that are consumed, and will affect the general structure of microbiota. Since plant-based foods contain many types of residue, microbiota will form a diverse structure with this diet. ${ }^{[2]}$ Beta glucan (oats and barley) and pectin (whole grain and apple) have high solubility and viscosity. They are also highly fermentable residue. ${ }^{[2]}$ Due to these attributes, beta glucan and pectin are favorable nutrients for nourishing gut microbiota.

In high-fiber diets, microbiota is well nourished and the amount of SCFAs formed as a result of fermentation increases. Due to the positive effects of SCFAs, fiber-rich nutrition improves health. ${ }^{[1]}$ Low-fiber diets reduce microbial diversity and also the amount of SCFAs. ${ }^{[1]}$

In order to regulate the gut microbiota, diet must be rich in carbohydrates (fructooligosaccharides, insulin, galactooligosaccharides, resistant starch) with prebiotic properties. $^{[2]}$

Table 2. The effect of natural and artificial elements on microbiota ${ }^{[2]}$

\begin{tabular}{lcccc}
\hline & Bifidobacteria & Bacteroides & Clostridia & Lactobacilli \\
\hline Glucose & $\uparrow$ & $\downarrow$ & & \\
Fructose & $\uparrow$ & $\downarrow$ & & \\
Sucrose & $\uparrow$ & $\downarrow$ & & \\
Lactose & $\uparrow$ & $\downarrow$ & $\downarrow$ & $\uparrow$ \\
Artificial sweetener & $\downarrow$ & $\uparrow$ & $\downarrow$ & $\downarrow$ \\
\hline
\end{tabular}


Table 3. Age-related changes in gut microbiota ${ }^{[7]}$

\begin{tabular}{|c|c|c|}
\hline Age-related changes in gut microbiota & Outcome & Mechanism \\
\hline Decreased biodiversity & $\begin{array}{l}\text { Increased prevalence of } C \text {. difficile-related } \\
\text { diarrhea }\end{array}$ & Decreased resistance to $C$. difficile \\
\hline Enterobactericeae proliferation & $\begin{array}{l}\text { Increased inflammatory response } \\
\text { Increased colon cancer metastasis }\end{array}$ & Increased endotoxin production \\
\hline Decreased butyrate production of bacteria & $\begin{array}{l}\text { Weakened colon epithelia } \\
\text { Increased inflammatory response } \\
\text { Increased colorectal cancer development }\end{array}$ & $\begin{array}{l}\text { Reduced protective and trophic effects of } \\
\text { butyrate in colon epithelium } \\
\text { Reduced antiinflammatory effects of butyrate } \\
\text { Reduced antineoplastic effects of butyrate }\end{array}$ \\
\hline Increased colonization and toxin production & Increased colorectal cancer development & $\begin{array}{l}\text { Impaired enterocyte cell cycle and growth } \\
\text { control and deoxyribonucleic acid damage }\end{array}$ \\
\hline
\end{tabular}

\section{2) Proteins}

Ammonia, amines, indole, phenols, sulfur compounds and organic acids emerge as a result of degradation and fermentation of proteins that are ingested by the diet and reach the colon without being digested. ${ }^{[1]}$

High protein diets are known to be associated with Bacteroides enterotype, as a result of observational studies of people in different geographies. ${ }^{[1]}$ If the consumption of red meat is high in the diet, the number of Bifidobacterium adolescentis is expected to decrease and the number of Bacteroidetes and Clostridia is expected to increase compared to when meat is not being consumed. ${ }^{[1]}$

Russel et al. ${ }^{[1]}$ found that a diet rich in protein and poor in carbohydrates caused a significant reduction in butyrate-producing microorganisms (Rosembria, Eubacterium rectale, etc.). In addition, high-protein diets increase proteolytic fermentation in the colon and the metabolites resulting from this fermentation create a risky environment for inflammation and colon cancer. ${ }^{[7]}$

\section{3) Fats}

Dietary fats affect the microbiota in three aspects: the amount of fat ingested, the type of fat ingested, and the effect of fat on secondary bile acid formation. ${ }^{[7]}$

High-fat diet significantly reduces microbiota diversity while increasing the number of Bilophila, Bacteroides, and Alistipes. In addition, studies on mice found that high-fat diet was associated with microorganisms producing propionate and acetate. Such a diet can affect inflammation associated with metabolic endotoxemia. ${ }^{[1]}$ High-fat diets have been found to significantly reduce the amount of fecal SCFAs as well as the number of Bifidobacteria compared to low-fat diets. ${ }^{[7]}$

Another experiment on mice observed dysbiosis and metabolic dysfunctions in mice fed with foods rich in saturated fatty acid, while mice fed with polyunsaturated fatty acid-rich fish oil had lactic acid produced by bacteria and no metabolic dysfunction..$^{7]}$

Fat-rich diets stimulate secondary bile acid secretion, and as a result, the amount of secondary bile acid increases in the colon. This phenomenon causes only microorganisms resistant to bile acids to multiply in the gut and drastically changes the microbiota profile. ${ }^{[7]}$ These changes cause increased intestinal permeability and endotoxin production resulting in increased lipopolysaccharide translocation and, consequently, increased levels of circulating lipopolysaccharides. This increase is called metabolic endotoxemia and plays an important role in physiopathology of diseases such as obesity, diabetes, and hypertension. ${ }^{[7]}$

\section{4) Phytochemicals}

Polyphenols and glucosinolates are the most important phytochemicals for gut microbiota. Polyphenols are commonly found in vegetable foods such as fruits, vegetables, tea, cocoa, and are usually in the form of glucosides in these foods. Most polyphenols reach the colon without being digested, where they are transformed into an active form, the aglycone form, undergoing degradation. ${ }^{[1,7]}$ 
Polyphenols have antimicrobial and bacteriostatic effects, which suppress pathogenic microorganisms. In addition, they facilitate proliferation of beneficial microorganisms with prebiotic properties. ${ }^{[7]}$ Bifidobacterium and Lactobacillus bacteria have been shown to be affected by dietary polyphenol intake. ${ }^{[1]}$ Furthermore, the bioactive components in tea stop the growth of Helicobacter pylori, ${ }^{[1]}$ Staphylococcus aureus, and E. Coli. ${ }^{[1]}$

\section{5) Vitamins}

Vitamins are essential nutrients for living metabolism. There are two ways to satisfy the need for vitamins in humans. The first is the exogenous intake of vitamins, while the second is the production of certain vitamins ( $\mathrm{B}$ group and $\mathrm{K}$ vitamins) by microbiota and their absorption from the colon. ${ }^{[3]}$ Vitamins are essential to the regulation of general metabolism. Microbiota is the main source of certain vitamins.

In an experimental study on humans, microbiota suppressive drugs were given to one group and not to the other. Both groups were provided with a diet insufficient in vitamin $\mathrm{K}$ for four weeks. While there was no vitamin deficiency in the drug-free group, a significant decrease in plasma prothrombin levels was observed in the drug-using group. ${ }^{[1]}$

Bacteroidetes, Fusobacteria, and Protobacteria groups can produce vitamins B2 and B7. In addition, all Fusobacteria can produce vitamin $\mathrm{B} 12$. Bacteroidetes generally produce the most vitamin $B{ }^{[1]}$

While Vitamin A is important in formation of intestinal response against pathogens and antigens, vitamin $\mathrm{D}$ is essential to the intestinal immune system and the mucosal barrier. ${ }^{[3]}$

\section{6) Minerals}

Minerals act as regulators of metabolism and are important micronutrients for gut microbiota. Iron is effective in metabolic activity of gut microbiota. The amount of Lactobacillus significantly decreases in iron deficiency, but whether iron deficiency causes this decrease or whether this decrease causes iron deficiency is not yet known. ${ }^{[3]}$ Furthermore, not only does Lactobacillus decrease, but the numbers of Rosebria spp., E. rectale, and Bacteroides spp. species also significantly decrease. ${ }^{[3]}$

\section{THE SIGNIFICANCE OF ELDERLY AGE, PREBIOTICS, AND PROBIOTICS}

Probiotics are microorganisms that positively affect the health of the host they live in. ${ }^{[8]}$ In the neonatal gut, microorganisms of vaginal and dermal origin contracted from the mother during birth begin to colonize. Although these microorganisms are usually probiotics, there are a few pathogens among them. Probiotic-rich colonization is mandatory for a healthy immune system in newborns. ${ }^{[8]}$ The rise in chronic diseases is highly associated with decreased consumption of antioxidant and vegetable fibers. ${ }^{[8]}$

Our body has three main sources of probiotics:

1. Fermented dairy products

2. Foods with live probiotic bacteria added

3. Pharmacological products with live probiotic bacteria added ${ }^{[8]}$

According to the 2002 definition by the WHO, microorganisms that have beneficial effects on human and animal health are called probiotics. The probiotics must be:

- Adaptable to the intestinal flora

- Resistant to stomach and bile acid

- Colonize by adhering to the gastrointestinal wall

- Positively affect health ${ }^{[8]}$

- Stable during production and storage

- Able to secrete antimicrobial substances (such as bacteriocin)

- Non-pathogenic ${ }^{[1]}$

Probiotics are generally abundant in fermented dairy products such as yogurt, kefir, ale yeast, wine, vinegar, and pickles. Lactobacilli and Bifidobacteria are the most abundant groups in these foods. ${ }^{[8]}$ Studies on yogurt and kefir have shown that products containing probiotics increase the population of Bifidobacterium and lactic acid bacteria, galactosidase activity, and the amount of SCFAs, while reducing the number of Enterobacteria and Clostridia. ${ }^{[1]}$

Some of the functions of probiotics that make it difficult for pathogens to settle in the intestines include: lowering the intestinal $\mathrm{pH}$, secreting antimicrobial compounds against pathogens, competing with pathogens to bind to receptors, 
and preventing them from being trapped in the gut wall. They also reduce the survival chances of pathogens by consuming nondigestible foods. ${ }^{[8]}$

Prebiotics are nutritional compounds that selectively effect the function and activity of selectively fermentable microbiota, positively effecting the person's health. ${ }^{[1]}$ Prebiotics are a class of nutrients generally consisting of carbohydrates which are not digested throughout the digestive system and provide nourishment to microorganisms of the gut microbiota. ${ }^{[8]}$

Prebiotics allow the formation of SCFAs through fermentation in the colon. They also have beneficial effects on intestinal immunity and metabolic functions. The relationships achieved through the fermentation of residue improve the gut-associated lymphoid tissue (GALT). ${ }^{[1]}$ Prebiotics are abundant in fiber-rich foods (such as apples, bananas, barley, wheat), especially breast milk. ${ }^{[8]}$

When a dietary element contains both beneficial microorganisms (probiotics) and non-digestible substances (prebiotic) to nourish it (such as breast milk), these foods are called symbiotic foods. If the nutrient contains beneficial bacteria metabolit, it is called postbiotic nutrient. ${ }^{[8]}$

The aging process is dependent on many factors, including genetics and epigenetics. Pathophysiological changes in the gut microbiota are one of the most important factors that affect the aging process. ${ }^{[8]}$ Aging, as well as malfunctions in the physiology of the digestive system along with changes in nutritional habits, cause drastic changes in microbiota and disrupt the homeostatic balance between the microbiota and host. ${ }^{[8]}$ Many diseases are associated with microbiota during the aging process.

Gut microbiota, effective in the immune system and human metabolism, is thought to have the greatest impact on longevity of life. In people that live long, data has shown increased number of Prevotella, Firmicutes, and Ruminococeaceae group bacteria, decreased number of Lachnospiraceae, and more moderate increase in Bacteroidetes group bacteria. ${ }^{[9]}$

In elderly age, unnecessary use of antibiotics should be avoided, fiber-rich natural diet should be consumed, and probiotic support should be given. ${ }^{[9]}$ Some of the benefits of probiotic use in old age include: regulation of immune functions, reduced side effects of antibiotics, prevention of cancers, effective treatment of inflammatory bowel diseases. Since nutrition can be optimized, it is the easiest and most inexpensive method to preserve health in old age. ${ }^{[9]}$

In conclusion, human gut microbiota has important effects on human health. While it significantly prevents the colonization of harmful bacteria in the gut, it also acts as an essential regulator of the immune system. Most chronic diseases are thought to originate from disorders in the gut microbiota. Nutrition is one of the most important factors affecting human intestinal microbiota draws much needed attention as it is a regulable factor. Nutrition should be closely watched and diet should be enriched with probiotics and prebiotics, from the period of breast milk, the first dietary element, by parents, and later by the person's own self. While the Mediterranean diet seams to be the most appropriate diet for microbiota health, further studies are needed to make more definite recommendations.

\section{Declaration of conflicting interests}

The authors declared no conflicts of interest with respect to the authorship and/or publication of this article.

\section{Funding}

The authors received no financial support for the research and/or authorship of this article.

\section{REFERENCES}

1. Özdemir A, Demirel ZB. Beslenme ve mikrobiyota ilişkisi. J Biotechnol and Strategic Health Res 2017;1:25-33.

2. İpek KD, Yllmaz HÖ. Diyetin ve karbonhidrat içeriğginin mikrobiyotaya etkisi. Cumhuriyet Üniv Sağ Bil Enst Derg 2018;2:29-39.

3. Koçak T, Şanlıer N. Mikrobesin öğeleri ve mikrobiyota etkileșimi. GÜSBD 2017;6:290-302.

4. Güney R, Çınar N. Anne sütü ve mikrobiyota gelişimi. J Biotechinol \& Strategic Health Res 2017;1:17-24.

5. De Filippo C, Cavalieri D, Di Paola M, Ramazzotti M, Poullet JB, Massart S, et al. Impact of diet in shaping gut microbiota revealed by a comparative study in children from Europe and rural Africa. Proc Natl Acad Sci U S A 2010;107:14691-6.

6. Kurt ND, Okan Bakır B. Diyetin makro besin içeriğinin bağırsak mikrobiyotasına etkisi. Uluslararası Hakemli Beslenme Araştırmaları Dergisi 2018;5:17-42. 
7. Büyüktunçer Demirel Z. Beslenme ve mikrobiyota. TÜBA Mikrobiyota ve İnsan Sağlığı Sempozyumu Raporu; 2017. s. 37-45.

8. Yllmaz Alkan Ö. Yaşlılarda Sağlıklı Beslenme- probiyotikler. Ege TIP Dergisi 2015;54 Supp:16-21

9. Saka B. Yaşlanma, uzun yaşam ve mikrobiyota. Tüba Mikrobiyota ve İnsan Sağlığı Sempozyumu Raporu; 2017. s. $52-4$. 